\title{
A meeting of parallel paths for US fusion?
}

Colin Macilwain

\section{The United States is realigning its nuclear fusion programme to improve the integration of research into two rival approaches to fusion pow er: magnetic and inertial confinement. The tas $k$ is a challenging one.}

[WASHINGTON] A series of top-level reviews and scientific meetings will be carried out this year to try to find ways of integrating US research in magnetic and inertial confinement fusion. The move follows the country's withdrawal last year from the main global research collaboration aimed at achieving controlled nuclear fusion.

Magnetic confinement fusion has historically been supported in the United States by the Department of Energy's Fusion Energy Sciences programme, while inertial confinement fusion is supported by the department's nuclear weapons programme.

The Fusion Energy Sciences programme was sharply cut three years ago, as Congress appeared to lose patience with the feasibility of fusion as an energy source. This was followed last summer by US withdrawal from the International Thermonuclear Experimental Reactor (ITER) project, a global collaboration to build a test-bed for magnetic fusion (see Nature 394, 511-512; 1998).

But, although Congress has cooled on magnetic fusion, it has been willing to support a major new inertial confinement fusion experiment: the $\$ 1.5$ billion National Ignition Facility (NIF) at the Lawrence Livermore Laboratory in California.

At NIF, ignition is obtained by compressing a tiny pellet of hydrogen fuel with X-rays - the principle behind the hydrogen bomb. Congress supports this work because it will enable scientists to improve their understanding of nuclear weapons without testing.

Any possible energy implications are seen

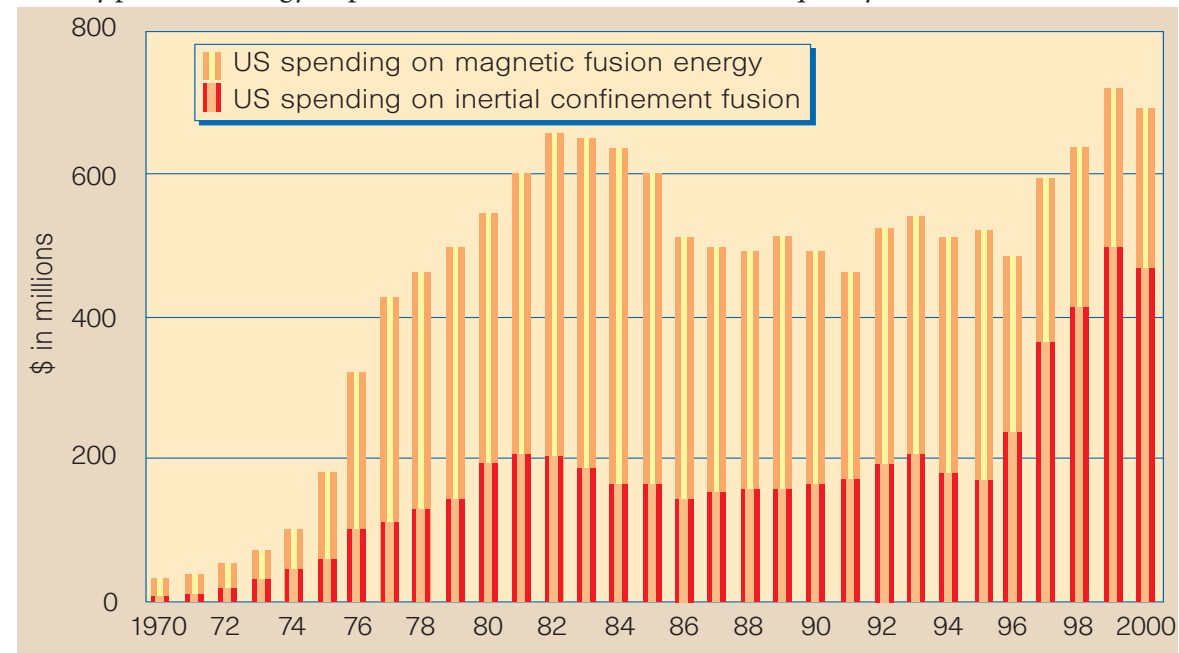

as an incidental benefit. The result, however, is a reversal of the respective positions of the two programmes over the past few years. In 1995 , the US spent $\$ 360$ million on the magnetic confinement fusion programme, and a little over half that amount on inertial confinement fusion. This year, it will spend $\$ 500$ million on inertial work and less than half of that on magnetic concepts.

The two programmes have many common technical needs, and the plasma physicists who lead them know each other well, having trained in the same places and attended scientific meetings together for years. But the rigid independence of the two strands of research, combined with the rapid changes in their respective fortunes, has raised questions about the balance of the overall programme as it now stands.

Last autumn, the Senate energy and water appropriations subcommittee asked the energy department to conduct a broad review of the entire fusion programme. The request produced a flurry of activity. Last month, for example, a sub-group of the Secretary of Energy's Advisory Board met in Washington to start a quick assessment of the "appropriate balance" between magnetic and inertial fusion.

The Fusion Energy Sciences Advisory Committee, chaired by John Sheffield of Oak Ridge National Laboratory in Tennessee, will report later this year on its priorities for the civilian science programme. The National Research Council is about to start an assessment of the quality of the research in the 